\title{
From bovels to
} todthbroushes

\section{Story by Caroline Holland.}

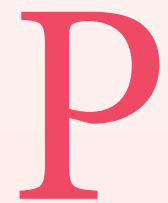

atients in hospital are regularly asked: 'Have you opened your bowels today?' But now, Urshla Devalia, Consultant in Paediatric Dentistry, pictured right above, wants medical staff in hospitals with a paediatric in-patient base, like Great Ormond Street Hospital where she works, to put greater emphasis on the question: 'Have you brushed your teeth today?'

A stay in hospital is an opportunity to make every contact count and for children to be taught about oral hygiene. She has patients whose future depends on the dental treatment she delivers. Seriously ill children with compromised immune systems or those who might need a life-saving heart operation or cancer treatment need a healthy mouth. Unless all dental decay and infection is eradicated, they will not be able to undergo the treatment they need to save their life. worries, but the mouth must be made healthy in order for the life-saving cardiac implant to be placed. The patient has broken teeth, with only 3 roots remaining where the back molars should be and these are surrounded by infection and bone loss. The roots must be surgically removed under a general anaesthetic and at the same time she must fill 14 holes in the remaining teeth. Because of the child's heart condition, a general anaesthetic is a high risk procedure. Ms Devalia had the difficult job of explaining to the parents that their child had to go through a risky dental operation before he could undergo his life-saving surgery. This is not an isolated case.

But now, nurses, health care assistants and doctors working at the hospital are to be trained by Ms Devalia and GOSH specialist dental nurse, Claire Fletcher, pictured left above, to help implement a pioneering new

\section{'NURSES, HEALTH CARE ASSISTANTS AND DOCTORS}

\section{WORKING AT THE HOSPITAL ARE TO BE TRAINED}

\section{BY MS DEVAUA AND GOSH SPECIAUST DENTAL}

\section{NURSE, CLAIRE FLETCHER, TO HELP IMPLEMENT}

\section{A PIONEERING NEW PROGRAMME CALLED}

\section{MINI MOUTHCARE MATTERS (MINI MCM).'}

One of her young patients has a rare congenital heart condition. Because the chambers of the heart are not fully formed, death can occur at any time. The solution is an operation to place a cardiac defibrillator which will kick into action if the heart fails. The patient's teeth should be the least of their programme called Mini Mouthcare Matters (Mini MCM).

In future, staff on paediatric wards will integrate dental health into the overall care they offer. For instance, they will ask young patients - or their parents/carers - if they have brought in a toothbrush for their

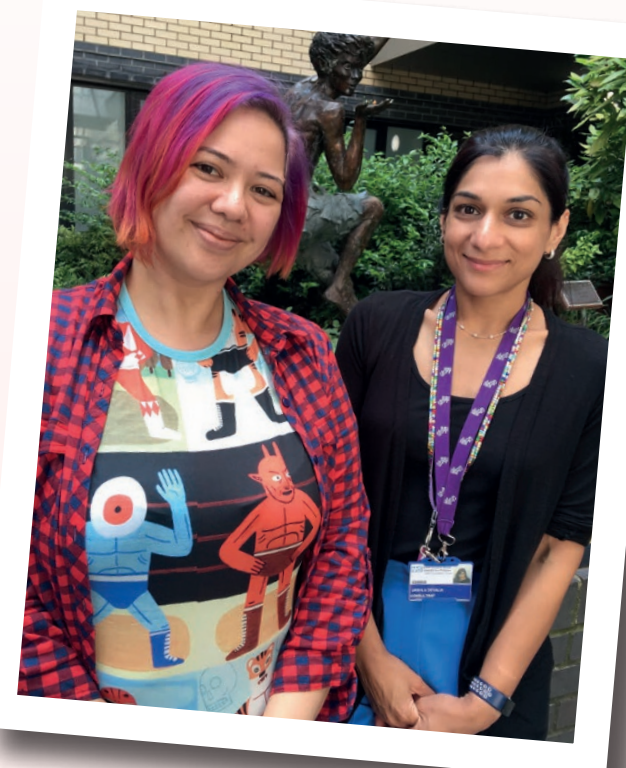

overnight stay and when they last saw a dentist. The programme is funded by Health Education England and GOSH will be working in collaboration with Birmingham Children's Hospital, Royal Manchester Children's Hospital and University College London Hospitals with a view to rolling the programme out nationally in 2019.

Ms Devalia aims to empower all healthcare professionals working in hospitals with paediatric wards to incorporate oral health into the care routine for those patients with a stay of more than 24 hours. Mini Mouthcare Matters should be applicable to any hospital with a paediatric in-patient base.

Ms Devalia said: 'Our young patients are already struggling with complex and serious conditions. It's vital that there is no risk of an infection in the mouth which could cause complications and delays to their treatment. By integrating dental hygiene into overall care, health professionals can take every opportunity to highlight how important a healthy mouth and teeth are to general well-being.'

Mini MCM was inspired by a programme dedicated to improving the oral care of older residents in hospital, care homes and community settings in the Kent, Surrey \& Sussex region. Ms Devalia acknowledged the advice and support of Mili Doshi, founder of MCM, and her team. Mili Doshi, a consultant in Special Care Dentistry, has recently been awarded an MBE in the Queen's Birthday Honours.

${ }^{*}$ Later in the year, BDJ Team will feature a 'Day in the Life of' article by dental nurse Claire Fletcher. 